\title{
Neoadjuvant brachytherapy and chemotherapy followed by radical surgery for stage IB2 and IIA cervical cancer: A retrospective comparison with chemoirradiation
}

\author{
YAOMEI MA ${ }^{1}$, GUILING ZHAO ${ }^{1}$, JI QI ${ }^{1}$, PEISONG SUN ${ }^{1}$, CAIYAN LIU $^{1}$, PENGPENG QU $^{1}$ and KAREN K.L. CHAN ${ }^{2}$ \\ ${ }^{1}$ Department of Gynecological Oncology, Tianjin Central Hospital of Obstetrics and Gynecology, \\ Nankai, Tianjin 300241; ${ }^{2}$ Department of Obstetrics and Gynecology, University of Hong Kong, \\ Queen Mary Hospital, Hong Kong SAR 999077, P.R. China \\ Received May 4, 2016; Accepted February 6, 2018
}

DOI: $10.3892 / \mathrm{mco} .2018 .1580$

\begin{abstract}
The aim of the present study was to assess the immediate and long-term results of preoperative brachytherapy and chemotherapy followed by radical surgery compared with those of standard chemoirradiation in patients with stage IB2-IIA cervical cancer. The medical records of 70 patients with stage IB2 and IIA cervical cancer who were treated between June 2006 and June 2010 were reviewed. The patients received either standard chemoirradiation (CRT) treatment $(n=20)$ or neoadjuvant brachytherapy with one cycle of chemotherapy followed by radical hysterectomy [operation (OT) group; n=50]. Further adjuvant chemoirradiation was administered to patients with high-risk disease. Early and late complications as well as survival were compared between the two groups. No serious operative complications occurred in the OT group. In the CRT group, the incidence of symptomatic vaginal stenosis, as well as that of proctitis and cystitis, was higher compared with that in the OT group (35 vs. $4 \%$ and 20 vs. $2 \%$, repectively). The median follow-up period was 52 months (range, 11-84 months). In the CRT group, the 3-year overall and disease-free survival rates were 95\% [95\% confidence interval (CI): 76.14-86.46] and 90\% (95\% CI: 59.94-73.66), respectively, whereas in the OT group, the respective rates were $90 \%$ (95\% CI: 72.93-83.07) and $90 \%$ (95\% CI: 71.84-82.96). In conclusion, the survival of patients with stage IB2-IIA cervical cancer treated with preoperative brachytherapy and chemotherapy followed by radical surgery was similar to that of patients treated with chemoirradiation, but with a more favorable side effect profile. Thus, this
\end{abstract}

Correspondence to: Professor Karen K.L. Chan, Department of Obstetrics and Gynecology, University of Hong Kong, Queen Mary Hospital, 102 Pokfulam Road, Hong Kong SAR 999077, P.R. China E-mail:kklchan@hku.hk

Key words: cervical cancer, radical hysterectomy, radiotherapy, survival, complications tri-modal treatment option requires further evaluation in prospective randomized studies.

\section{Introduction}

Cervical cancer is the second most common type of cancer worldwide and the leading cause of cancer-related mortality among women in developing countries (1). Although surgery is reserved for early-stage disease whereas locally advanced cervical cancer (LACC) is treated by chemoirradiation (CRT) (2-7), there is an increasing number of studies on multimodal treatments of LACC, including preoperative radiotherapy (RT) and chemotherapy followed by surgery (8-11). Different multimodality regimens have been suggested, including preoperative pelvic RT along with cisplatin and 5-fluorouracil (8-10), preoperative low-dose rate uterovaginal brachytherapy (9) and external pelvic CRT with low-dose cisplatin-based chemotherapy followed by brachytherapy and subsequent surgery (11). However, despite the promising results, severe side effects and a delay in definitive treatment remain a major concern. The aim of the present study was to review our experience with neoadjuvant treatment consisting of brachytherapy with one course of chemotherapy, which may be completed within 1-2 weeks, immediately followed by radical surgery. The short- and long-term outcomes of this regimen were compared with those of standard CRT in stage IB2 and IIA patients.

The present study was approved by the institutional Ethics Review Board of Tianjin Central Hospital of Obstetrics and Gynecology

\section{Patients and methods}

Patients and treatments. The present study retrospectively reviewed the medical records of all patients clinically staged as IB2 or IIA according to the International Federation of Gynecology and Obstetrics (FIGO) 2000 criteria (12) at Tianjin Central Hospital of Obstetrics and Gynecology (Nankai, China) over a 4-year period (June 2006-June 2010). All the patients were clinically staged by two gynecological oncologists and cervical biopsies were taken for histological 
confirmation. The patients were allocated to either the CRT group or the operation (OT) group, mainly based on age and overall medical condition. Patients in the CRT group were treated with external pelvic RT and brachytherapy with concomitant chemotherapy. A dose of 50 Gy in 26-30 fractions was delivered to the pelvis by ${ }^{60} \mathrm{Co}$ external-beam RT (ERT). ${ }^{192} \mathrm{Ir}$ intracavity brachytherapy was delivered once a week during ERT to a total dose of 30-50 Gy at point A (defined as the point of crossing of the uterine artery and ureter). ERT was omitted on the day of intracavity brachytherapy. Chemotherapy was started at the beginning of ERT. Patients either received 3-4 cycles of three-weekly cisplatin at $50 \mathrm{mg} / \mathrm{m}^{2}$ together with 5 -fluorouracil $20 \mathrm{mg} / \mathrm{kg}$ for 4 consecutive days, or cisplatin at $50 \mathrm{mg} / \mathrm{m}^{2}$ and one dose of paclitaxel $\left(135-175 \mathrm{mg} / \mathrm{m}^{2}\right)$, based on the gynecologist's preference and the financial situation of the patient. Patients in the OT group first underwent preoperative vaginal brachytherapy twice within 1 week. The median delivered dose to the clinical target volume $(1 \mathrm{~cm}$ from the radioactive source) was 20-24 Gy. One cycle of chemotherapy with bleomycin, vincristine and cisplatin ( $2 \mathrm{mg}$ vincristine on the first day and a total of $50 \mathrm{mg} / \mathrm{m}^{2}$ cisplatin in five doses over 5 consecutive days, and $15 \mathrm{mg}$ bleomycin on alternate days administered in three doses) was administered either during the brachytherapy week or immediately thereafter. After neoadjuvant treatment, the patients were examined by the same two gynecologists to assess clinical response. Patients with a tumour size of $<4 \mathrm{~cm}$ and clinical tumor improvement underwent radical hysterectomy (RH) and systematic pelvic lymph-node dissection within 3 weeks of neoadjuvant treatment. Patients not fulfilling these criteria were re-assigned to the CRT group. Postoperatively, patients with high-risk factors, including pelvic lymph node metastasis, positive resection margin or parametrial invasion, received adjuvant concurrent CRT. Patients who had $\geq 2$ intermediate risk factors, including deep stromal invasion, defined as invasion into the cervical wall by more than half of its thickness, lymphovascular space invasion, or a tumor size of $\geq 4 \mathrm{~cm}$, received postoperative RT alone. After completion of treatment, patients were followed up every 3 months for the first 2 years, then every 6 months for the following 3 years, and once a year thereafter, with clinical examination, cervical or vault smear, and assessment of squamous cell carcinoma antigen (SCC) antigen levels.

Statistical analysis. SPSS software version 13.0 (SPSS, Inc., Chicago, IL, USA) was used for all statistical analyses. The $\chi^{2}$ test or Fisher's exact test were used to compare discrete variables among groups. Disease-free survival (DFS) was calculated from the date of surgery or the date of completed CRT to the date of relapse or the date of the last follow-up, and overall survival $(\mathrm{OS})$ was calculated from the date of diagnosis to the date of death or the date of the last follow-up. Medians and life tables were computed using the product limit estimate by the Kaplan-Meier method, and the log-rank test was used to assess statistical significance.

\section{Results}

Patient characteristics. Between June 2006 and June 2010, a total of 70 patients were diagnosed with stage IB2 or IIA cervical cancer at Tianjin Central Hospital of Obstetrics and Gynecology
(Nankai, China). The OT group included 50 patients and the CRT group 20 patients. The median follow-up period was 48 months (range, 30-72 months) for the CRT group and 54 months (range, 11-84 months) for the OT group.

The patient and tumor characteristics, including histological type, stage and grade, are summarized in Table I. There were no significant differences in age, histology or grade between the two groups; however, the OT group included a higher number of stage IB2 patients compared with the CRT group.

Surgery. A total of 50 patients underwent Piver type III RH and pelvic lymphadenectomy. Bilateral salpingo-oophorectomy was performed in 9 patients with adenocarcinoma of the cervix and in 16 patients with SCC (menopause), 2 patients with adenosquamous carcinoma and 2 patients with sarcoma. Ovarian transposition was performed in 21 patients with SCC. Pelvic lymphadenectomy included the external iliac, internal iliac, obturator and common iliac lymph nodes.

The median interval between preoperative therapy and surgery was 25.8 days (range, 15-50 days). Intra- and postoperative complications were evaluated in all surgical patients. The median operative time was $195 \mathrm{~min}$ (range, 110-285 min) and the median blood loss was $583 \mathrm{ml}$ (range, 200-1,400 ml). Of the 50 patients, 14 (28\%) suffered an estimated blood loss of $>800 \mathrm{ml}$ during surgery and were transfused. The median duration of hospital stay was 7 days (range, 4-18 days). The surgical specimens of 7 patients (14\%) contained no tumor following neoadjuvant CRT, while residual tumors were found in the surgical specimens of 43 patients (86\%). Among patients with residual tumors, 42 (98\%) had a residual tumor in the cervix and 1 patient (2\%) had a residual tumor in the vagina alone. Among all patients in the OT group, 12 (24\%) had pelvic lymph node involvement, of whom 2 (4\%) also had parametrial invasion. The pathological results of the patients in the OT group are summarized in Table II.

Postoperative therapy. A total of 26 patients received postoperative therapy. A total of 13 patients (26\%) with pelvic lymph node involvement, parametrial invasion and positive vaginal margin received concurrent CRT, among whom 6 received cisplatin $\left(50 \mathrm{mg} / \mathrm{m}^{2}\right)$ with 5 -fluorouracil $(20 \mathrm{mg} / \mathrm{kg})$ daily for 4 days, repeated twice with a 21-day interval, 5 received paclitaxel $\left(135-175 \mathrm{mg} / \mathrm{m}^{2}\right)$ and cisplatin $\left(50-60 \mathrm{mg} / \mathrm{m}^{2}\right)$, and 2 patients were treated with docetaxel $\left(60 \mathrm{mg} / \mathrm{m}^{2}\right)$ and carboplatin (area under the curve 4) for 4-6 cycles. Standard-field ERT was administered for 4-6 weeks after surgery to a total dose of $40 \mathrm{~Gy}$. Among these patients, 1 (2\%) received a parametrial boost to a total of $50 \mathrm{~Gy}$ due to a positive vaginal margin and 2 patients (4\%) received extended-field ERT due to common iliac lymph node involvement. All the patients completed their prescribed treatment.

Additional ERT was administered to 13 patients (26\%) with $\geq 2$ intermediate risk factors. The median time interval between surgery and RT was 40 days (range, 30-46 days). The median delivered dose to the pelvis was 40 Gy (range, $30-50 \mathrm{~Gy}$ ) for the standard field; 2 of those patients did not complete the treatment against doctors' recommendations.

Complications. Early complications, defined as those occurring within 2 months after surgery, developed in 9 patients 
Table I. Characteristics of patients in the two groups.

\begin{tabular}{lccc}
\hline Characteristics & $\begin{array}{c}\text { CRT, } \mathrm{n} \\
(\mathrm{n}=20)\end{array}$ & $\begin{array}{c}\text { OT, } \mathrm{n} \\
(\mathrm{n}=50)\end{array}$ & P-value \\
\hline Median age (years) & 47.5 & 43.8 & 0.09 \\
Histology & & & 0.19 \\
Squamous cell carcinoma & 20 & 37 & \\
Adenocarcinoma & 0 & 9 & \\
Adenosquamous carcinoma & 0 & 2 & \\
Other & 0 & 2 & \\
Stage & & & $<0.01$ \\
IB2 & 3 & 33 & \\
IIA & 17 & 17 & \\
Grade & & & 0.10 \\
I & 0 & 9 & \\
II & 12 & 21 & \\
III & 8 & 20 & \\
\hline
\end{tabular}

CRT, chemoradiotherapy group; OT, operation group.

Table II. Pathology results of patients in the operation group $(\mathrm{n}=50)$.

\begin{tabular}{lc}
\hline Pathology result & $\mathrm{n}(\%)$ \\
\hline Residual tumor & $43(86)$ \\
Squamous cell carcinoma & 31 \\
Adenocarcinoma & 8 \\
Adenosquamous carcinoma & 2 \\
Other (sarcoma) & 2 \\
Parametrial invasion & $2(4)$ \\
Positive lymph nodes & $12(24)$ \\
Positive vaginal margin & $1(2)$ \\
$\geq 2$ intermediate risk factors & $23(46)$ \\
Deep stromal invasion & $20(40)$ \\
Lymphovascular space invasion & $13(26)$ \\
Tumor size $\geq 4$ cm & $42(84)$ \\
\hline
\end{tabular}

(18\%). Among those, 4 patients had fever $>38^{\circ} \mathrm{C}$ for $>2$ days, 4 patients had urine retention requiring catheterization for $>2$ weeks, and 1 patient had thrombophlebitis. As regards late complications, the CRT group exhibited a higher frequency of symptomatic vaginal stenosis, as well as proctitis and cystitis ( 35 vs. $4 \%$ and 20 vs. $2 \%$, respectively) compared with the OT group. Grade 3-4 myelosuppression and lymphedema were similar between the two groups. The late complications of the CRT and OT groups are listed in Table III.

A subgroup analysis of stage IIA patients $(n=17$ in each group) revealed that the rate of complications in the CRT group was higher compared with that in the OT group $(\mathrm{P}=0.037$; Table IV).

Follow-up. As of June 2013, the median duration of follow-up was 52 months (range, 11-84 months) in the entire patient cohort, 49 months (range, 30-72 months) in the CRT group and 54 months (range, 11-84 months) in the OT group. All the patients were followed up for at least 3 years. In the OT group, 5 patients $(10 \%)$ developed recurrence. Among them, 1 patient had a vaginal recurrence after 29 months. One patient with cervical sarcoma had pelvic metastasis within 2 years after treatment. Another patient had parametrial recurrence 26 months later and succumbed to disease progression; this patient exhibited a poor response to neoadjuvant CRT and did not complete the postoperative ERT. One patient suffered a metastatic relapse 6 months later, affecting the pelvis and lung, and succumbed to the disease within 1 year; this patient exhibited parametrial and pelvic lymph node involvement. Another patient developed recurrence near the ureter and sigmoid colon after 5 months and succumbed to the disease; this patient had not received any postoperative therapy due to the absence of risk factors. The 3-year overall and disease-free survival rates in the OT group were 90\% [95\% confidence interval (CI): 72.93-83.07] and 90\% (95\% CI: 71.84-82.96), respectively (Figs. 1 and 2).

In the CRT group, 2 patients $(10 \%)$ suffered a relapse: In 1 patient the relapse affected the pelvic lymph nodes, while the other patient developed extensive disease (affecting the pelvic lymph nodes, lung and supraclavicular lymph nodes). In the CRT group, the 3-year overall survival and disease-free survival rates were $95 \%$ (95\% CI: 76.14-86.46) and $90 \%$ (95\% CI: 59.94-73.66), respectively (Figs. 1 and 2).

In the subgroup of patients with stage IIA disease, 1 patient in the CRT group and 2 patients in the OT group suffered a recurrence and succumbed to the disease. There was no statistically significant difference in survival between the two groups.

\section{Discussion}

Early-stage cervical cancer is commonly treated by surgery alone. For patients with FIGO stage IB2-IVA disease, also referred to as LACC, the preferred therapy is concurrent CRT. The use of a three-modality treatment, including RT, chemotherapy and surgery, has been reported as early as $1994(13,14)$. Recent studies on preoperative chemotherapy and/or RT have shown promising results, including better local control and overall survival, while reducing short- and long-term complications, resulting in a better quality of life $(8,11,15-19)$. Another advantage of neoadjuvant treatment is that the response in the post-neoadjuvant biopsy sample may also identify patients who are more suitable for definitive CRT rather than surgery. For example, patients insensitive to neoadjuvant CRT were not considered suitable for $\mathrm{RH}$ due to the higher likelihood of high-risk factors, as they would have required further adjuvant chemotherapy or RT, which may have increased the occurrence of complications.

The present study used a three-modality treatment strategy comprising RT, chemotherapy and surgery for patients with stage IB2-IIA cervical cancer in an attempt to improve the overall and disease-free survival and reduce the requirement for adjuvant therapy, thereby minimizing complications.

The overall survival and disease-free survival rates of the present study were similar between the two groups. The 3-year disease-free survival rate of $90 \%$ and OS rate of 
Table III. Late complications in the chemoradiotherapy (CRT) and operation (OT) groups.

\begin{tabular}{lccr}
\hline Late complications & $\begin{array}{c}\text { CRT, } \mathrm{n}(\%) \\
(\mathrm{n}=20)\end{array}$ & $\begin{array}{c}\text { OT, } \mathrm{n}(\%) \\
(\mathrm{n}=50)\end{array}$ & P-value \\
\hline Grade 3-4 myelosuppression & $2(10)$ & $2(4)$ & 0.572 \\
Proctitis and cystitis & $5(20)$ & $1(2)$ & 0.006 \\
Ureterohydronephrosis & $0(0)$ & $1(2)$ & 1.000 \\
Lymphedema & $2(10)$ & $3(6)$ & 0.619 \\
Symptomatic vaginal stenosis & $7(35)$ & $2(4)$ & 0.002 \\
\hline
\end{tabular}

Table IV. Late complications of stage IIA patients in the chemoradiotherapy (CRT) and operation (OT) groups.

\begin{tabular}{lllr}
\hline Late complications & $\begin{array}{c}\text { CRT, } \mathrm{n}(\%) \\
(\mathrm{n}=17)\end{array}$ & $\begin{array}{c}\text { OT, } \mathrm{n}(\%) \\
(\mathrm{n}=17)\end{array}$ & P-value \\
\hline Grade 3-4 myelosuppression & $1(5.9)$ & $1(5.9)$ & 0.037 \\
Proctitis and cystitis & $3(17.6)$ & $0(0)$ & \\
Ureterohydronephrosis & $0(0)$ & $0(0)$ \\
Lymphedema & $1(5.9)$ & $2(11.8)$ & $1(5.9)$ \\
Symptomatic vaginal stenosis & $5(29.4)$ & & \\
\hline
\end{tabular}

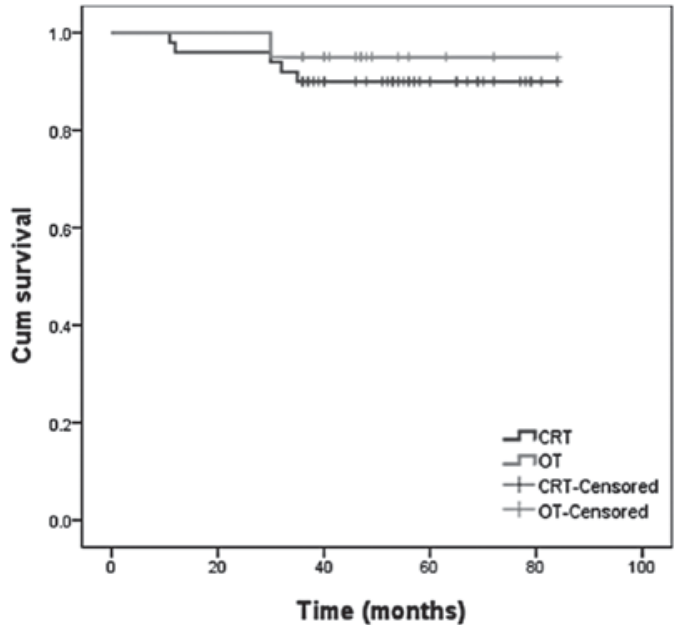

Figure 1. Overall survival curves for patients in the CRT and OT groups CRT, chemoradiotherapy group; OT, operation group; Cum, cumulative.

$91 \%$ observed in the entire cohort were encouraging when compared with the survival rate of $83 \%$ reported by previous studies (20-23). The results of the present study were also comparable to those of Ferrandina et al (8), who achieved local and distant control rates of 93 and $92 \%$, respectively, in patients with stage IB2-II over a similar follow-up time. The recurrence rate in the present study was $10 \%$ in each group. This result is comparable with that of a previous study, which reported a local recurrence rate of $16 \%$ in patients with stage IB and II disease treated with RT alone $(n=171)$ or surgery $(n=172)$ (22). At present, the study by Landoni et al (22) is the only single randomized controlled trial on patients with early-stage cervical cancer comparing RH followed by tailored adjuvant therapy with primary RT.

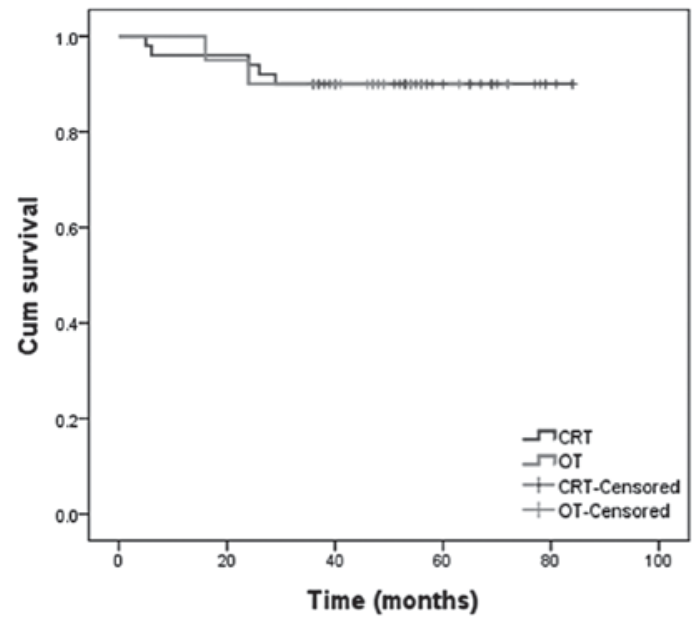

Figure 2. Disease-free survival curves for patients in the CRT and OT groups. CRT, chemoradiotherapy group; OT, operation group; Cum, cumulative.

With regard to the possible worsening of survival and recurrence rates with a longer follow-up period, it is important to consider that $88 \%$ of recurrences/progressions in the present study occurred within the first 2 years, which is in accordance with previous reports $(8,24)$. In the present study, all the patients were followed up for at least 3 years and $>70 \%$ of patients were followed up for $>4$ years.

The complications were similar between the CRT and OT groups in terms of grade 3-4 myelosuppression (10 vs. 4\%, respectively), ureterohydronephrosis (0 vs. $2 \%$, respectively) and lymphedema (10 vs. $6 \%$, respectively). However, in the CRT group, symptomatic vaginal stenosis, as well as proctitis and cystitis, were significantly more frequent compared with the OT group ( 35 vs. $4 \%$ and 20 vs. $2 \%$, respectively). Landoni et al (22) reported late complications in $16 \%$ of the 
patients treated exclusively with RT and in $29 \%$ of those treated with surgery and adjuvant RT. Among the patients with stage IB2 tumors, 84\% required adjuvant RT due to high-risk characteristics. By contrast, in the present study, only $50 \%$ of the patients in the OT group required adjuvant RT. The lower number of patients requiring adjuvant RT in the present study may have been due to the reduction of risk factors by preoperative CRT. In the present study, preoperative CRT included only one cycle of cisplatin, vinblastine and bleomycin chemotherapy and vaginal brachytherapy (20-24 Gy), which had few complications.

Various neoadjuvant treatments, including chemotherapy alone, RT alone and CRT, have been applied to patients with LACC (8-11,25-27). In the present study, neoadjuvant CRT was used. However, instead of the commonly used ERT, brachytherapy was used in the OT group, as it has been shown to provide local control with fewer complications (28-30).

The use of neoadjuvant chemotherapy has also been reported. Compared with RT treatment alone, the 5-year overall survival rate of patients subjected to neoadjuvant chemotherapy followed by surgery was $15 \%$ increased (25). A Cochrane meta-analysis revealed that progression-free survival was significantly improved by neoadjuvant chemotherapy followed by surgery compared with surgery alone; however, there was no overall survival benefit (27).

The aim of preoperative brachytherapy and chemotherapy is to decrease the size of the tumor in order to improve the feasibility of the operation. RT decreases the size of most tumors and facilitates resection with clear margins, while preoperative chemotherapy may decrease high-risk factors, such as lymphovascular involvement, reducing the requirement for adjuvant therapy and thereby minimizing the occurrence of complications (31). In the present study, the median time from neoadjuvant treatment to surgery was 25 days, which did not cause any significant delay to definitive treatment.

Radical surgery after CRT has been shown to reduce the rate of local recurrence $(18,32)$ and to improve the prognosis for patients with bulky residual tumors (33). Pathological assessment of surgical specimens allows for the identification of patients with poor response to CRT and also provides important prognostic information to guide the adjuvant therapy $(14,32,33)$.

In the present study, neoadjuvant CRT with brachytherapy followed by RH achieved good control of stage IB2 and IIA cervical cancer accompanied with a low incidence rate of complications compared with CRT alone. Although the present study was a retrospective analysis of a relatively small patient cohort and the chemotherapy regimen was somewhat heterogenous, it is one of few studies using brachytherapy instead of ERT for neoadjuvant CRT $(9,34,35)$. There were no significant differences in survival between the OT and CRT groups. A possible limitation of the present study was that the median follow-up duration was only 52 months. However, since most recurrences occurred within the first 24 months, a longer follow-up may yield the same outcome. Another limitation of the present study was the significant difference in stage distribution between the two groups. A subgroup analysis of stage IIA patients also demonstrated that the complication rates were lower in the OT group. However, there was no difference in OS between the OT and CRT groups.
In conclusion, the present study demonstrated that neoadjuvant CRT may reduce the tumor volume in a proportion of stage IB2-IIA patients, enabling them to undergo definitive surgery rather than definitive CRT, with a reduced incidence of long-term complications and similar overall survival compared with patients receiving CRT alone. A larger randomized trial with a standardized chemotherapy regimen and longer follow-up is required to confirm these results.

\section{References}

1. Jemal A, Bray F, Center MM, Ferlay J, Ward E and Forman D: Global cancer statistics. CA Cancer J Clin 61: 69-90, 2011.

2. Keys HM, Bundy BN, Stehamn FB, Muderspach LI, Chafe WE, Suggs CL III, Walker JL and Gersell D: Cisplatin, radiation and adjuvant hysterectomy compared with radiation and adjuvant hysterectomy for bulky stage IB cervical carcinoma. N Engl J Med 340: 1154-1161, 1999.

3. Morris M, Eifel PJ, Lu J, Grigsby PW, Levenback C, Stevens RE, Rotman M, Gershenson DM and Mutch DG: Pelvic radiation with concurrent chemotherapy compared with pelvic and para-aortic radiation for high risk cervical cancer. N Engl J Med 340: 1137-1143, 1999.

4. Whitney CW, Sause W, Bundy BN, Malfetano JH, Hannigan EV, Fowler WC Jr, Clarke-Pearson DL and Liao SY: Randomized comparison of fluorouracil plus cisplatin versus hydroxyurea as an adjunct to radiation therapy in stages IIB-IVA carcinoma of the cervix with negative para-aortic lymph nodes. A Gynecologic Oncology Group and Southwest Oncology Group Study. J Clin Oncol 17: 1339-1348, 1999.

5. Rose PG, Bundy BN, Watkins EB, Thigpen JT, Deppe G, Maiman MA, Clarke-Pearson DL and Insalaco S: Concurrent cisplatin-based radiotherapy and chemotherapy for locally advanced cervical cancer. N Engl J Med 340: 1144-1153, 1999.

6. Peters WA III, Liu PY, Barrett RJ II, Stock RJ, Monk BJ, Berek JS, Souhami L, Grigsby P, Gordon W Jr and Alberts DS: Concurrent chemotherapy and pelvic radiation therapy compared with pelvic radiation therapy alone as adjuvant therapy after radical surgery in high-risk early-stage cancer of the cervix. J Clin Oncol 18: 1606-1613, 2000.

7. Pearcey R, Brundage M, Drouin P, Jeffrey J, Johnston D, Lukka H, MacLean G, Souhami L, Stuart G and Tu D: Phase III trial comparing radical radiotherapy with and without cisplatin chemotherapy in patients with advanced squamous cell cancer of the cervix. J Clin Oncol 20: 966-972, 2002.

8. Ferrandina G, Legge F, Fagotti A, Fanfani F, Distefano M, Morganti A, Cellini N and Scambia G: Preoperative concomitant chemoradiotherapy in locally advanced cervical cancer: Safety, outcome and prognostic measures. Gynecol Oncol 107 (1 Suppl 1): S127-S132, 2007.

9. Ngô C, Alran S, Plancher C, Fourchotte V, Petrow P, Campitelli M, Batwa S, Sastre X, Salmon RJ and de la Rochefordière A; Institut Curie Gynaecological Cancer Study Group coordinated by P.Cottu: Outcome in early cervical cancer following pre-operative low dose rate brachytherapy: A ten-year follow up of 257 patients treated at a single institution. Gynecol Oncol 123: 248-252, 2011.

10. Ferrandina G, Margariti PA, Smaniotto D, Petrillo M, Salerno MG, Fagotti A, Macchia G, Morganti AG, Cellini N and Scambia G: Long-term analysis of clinical outcome and complications in locally advanced cervical cancer patients administered concomitant chemoradiation followed by radical surgery. Gynecol Oncol 119: 404-410, 2010.

11. Classe JM, Rauch P, Rodier JF, Morice P, Stoeckle E, Lasry S and Houvenaeghel G; Groupe des Chirurgiens de Centre de Lutte Contre le Cancer (GCCLCC): Surgery after concurrent chemoradiotherapy and brachytherapy for the treatment of advanced cervical cancer: Morbidity and outcome: Results of a multicenter study of the GCCLCC. Gynecol Oncol 102: 523-529, 2006.

12. Benedet JL, Bender H, Jones H, 3rd, Ngan HY and Pecorelli S: FIGO staging classifications and clinical practice guidelines in the management of gynecologic cancers. FIGO Committee on Gynecologic Oncology. Int J Gynaecol Obstet 70: 209-262, 2000.

13. Resbeut M, Cowen D, Viens P, Noirclerc M, Perez T, Gouvernet J, Delpero JR, Gamerre M, Boubli L and Houvenaeghel G: Concomitant chemoradiation prior to surgery in the treatment of advanced cervical carcinoma. Gynecol Oncol 54: 68-75, 1994. 
14. Jurado M, Martínez-Monge R, García-Foncillas J, Azinovic I, Aristu J, López-García G and Brugarolas A: Pilot study of concurrent cisplatin, 5-fluorouracil, and external beam radiotherapy prior to radical surgery + -- intraoperative electron beam radiotherapy in locally advanced cervical cancer. Gynecol Oncol 74: 30-37, 1999.

15. Mancuso S, Smaniotto D, Benedetti Panici P, Favale B, Greggi S, Manfredi R, Margariti PA, Morganti AG, Scambia G, Tortoreto F, et al: Phase I-II trial of preoperative chemoradiation in locally advanced cervical carcinoma. Gynecol Oncol 78: 324-328, 2000

16. Distefano M, Ferrandina G, Smaniotto D, Margariti AP Zannoni G, Macchia G, Manfredi R, Mangiacotti MG, Cellini N and Scambia G: Concomitant radiochemotherapy plus surgery in locally advanced cervical cancer: Update of clinical outcome and cyclooxygenase- 2 as predictor of treatment susceptibility. Oncology 67: 103-111, 2004.

17. Mariagrazia D, Anna F, Gabriella F, Francesco F, Daniela S, Giuseppe D, Alessio M and Giovanni S: Preoperative chemoradiotherapy in locally advanced cervical cancer: Long-term outcome and complications. Gynecol Oncol 99 (3 Suppl 1): S166-S170, 2005.

18. Motton S, Houvenaeghel G, Delannes M, Querleu D, Soulé-Tholy M, Hoff $\mathrm{J}$ and Lèguevaque $\mathrm{P}$ : Results of surgery after concurrent chemoradiotherapy in advanced cervical cancer: Comparison of extended hysterectomy and extrafascial hysterectomy. Int J Gynecol Cancer 20: 268-275, 2010.

19. Touboul C, Uzan C, Mauguen A, Gouy S, Rey A, Pautier P, Lhommé C, Duvillard P, Haie-Meder C and Morice P: Prognostic factors and morbidities after completion surgery in patients undergoing initial chemoradiation therapy for locally advanced cervical cancer. Oncologist 15: 405-415, 2010

20. Quinn MA, Benedet JL, Odicino F, et al: Carcinoma of the cervix uteri. FIGO 26th annual report on the results of treatment in gynaecological cancer. Int J Gynaecol Obstet 95: S43-S103, 2006.

21. Green JA, Kirwan JM, Tierney JF, Symonds P, Fresco L, Collingwood M and Williams CJ: Survival and recurrence after concurrent chemotherapy and radiotherapy for cancer of the uterine cervix: A systematic review and meta-analysis. Lancet 358: 781-786, 2001 .

22. Landoni F, Maneo A, Colombo A, Placa F, Milani R, Perego P, Favini G, Ferri L and Mangioni C: Randomised study of radical surgery versus radiotherapy for stage Ib-IIa cervical cancer. Lancet 350: 535-540, 1997.

23. Keys HM, Bundy BN, Stehman FB, Muderspach LI, Chafe WE Suggs CL III, Walker JL and Gersell D: Cisplatin, radiation and adjuvant hysterectomy compared with radiation and adjuvant hysterectomy for bulky stage IB cervical cancer. N Engl J Med 340: 1154-1161, 1999.
24. Houvenaeghel G, Lelievre L, Gonzague-Casabianca L, Buttarelli M, Moutardier V, Goncalves A and Resbeut M: Long-term survival after concomitant chemoradiotherapy prior to surgery in advanced cervical carcinoma. Gynecol Oncol 100: 338-343, 2006.

25. Dueñas-Gonzalez A, Cetina L, Mariscal I and de la Garza J: Modern management of locally advanced cervical cancer. Cancer Treat Rev 29: 389-399, 2003.

26. Moore DH: Treatment of stage IB2 (bulky) cervical carcinoma. Cancer Treat Rev 29: 401-406, 2003.

27. Rydzewska L, Tierney J, Vale CL and Symonds PR: Neoadjuvant chemotherapy plus surgery versus surgery for cervical cancer. Cochrane Database Syst Rev: CD007406, 2010.

28. Nori D, Dasari N and Allbright RM: Gynecologic brachytherapy I: Proper incorporation of brachytherapy into the current multimodality management of carcinoma of the cervix. Semin Radiat Oncol 12: 40-52, 2002.

29. Nag S, Chao C, Erickson B, Fowler J, Gupta N, Martinez A and Thomadsen B; American Brachytherapy Society: The American Brachytherapy Society for low-dose-rate brachytherapy for carcinoma of the cervix. Int J Radiat Oncol Biol Phys 52: 33-48, 2002.

30. Logsdon MD and Eifel PJ: Figo IIIB squamous cell carcinoma of the cervix: An analysis of prognostic factors emphasizing the balance between external beam and intracavitary radiation therapy. Int J Radiat Oncol Biol Phys 43: 763-775, 1999.

31. Hu JL, Wu LY, Li N, Li XG, Huang MN and Zhang R: Comparative analysis of neoadjuvant therapies in stage $\mathrm{Ib} 2$ and IIa2 cervical carcinoma. Chin J Obstet Gynecol 47: 452-457, 2012 (In Chinese).

32. Keys HM, Bundy BN, Stehman FB, Okagaki T, Gallup DG, Burnett AF, Rotman MZ and Fowler WC Jr; Gynecologic Oncology Group: Radiation therapy with and without extrafascial hysterectomy for bulky stage IB cervical carcinoma: A randomized trial of the Gynecologic Oncology Group. Gynecol Oncol 89: 343-353, 2003.

33. Houvenaeghel G, Lelievre L, Buttarelli M, Jacquemier J, Carcopino X, Viens P and Gonzague-Casabianca L: Contribution of surgery in patientswith bulky residual disease after chemoradiation for advanced cervical carcinoma. Eur J Surg Oncol 33: 498-503, 2007.

34. de la Rochefordiere A, Kirova Y, Alran S, et al: Pre-operative concomitant radio-chemotherapy in Bulky carcinoma of the cervix: a single institution study. Clin Med Oncol 2: 227-236, 2008

35. Pearcey RG, Peel KR, Thorogood J, et al: The value of pre-operative intracavitary radiotherapy in patients treated by radical hysterectomy and pelvic lymphadenectomy for invasive carcinoma of the cervix. Clin Radiol 39: 95-98, 1988. 